\title{
Is serum angiotensin converting enzyme level a useful non-invasive marker for liver fibrosis in patients with chronic hepatitis $\mathbf{C}$ ?
}

TARIK AKAR ${ }^{1 *}$ (1)

Study conducted at the Department of Gastroenterology, School of Medicine, Bülent Ecevit University, Zonguldak, Turkey

Article received: $7 / 4 / 2017$ Accepted for publication: $7 / 17 / 2017$ *Correspondence: Address: Yesil Mah. Aksemsettin Cad. Tersane Yapı Koop. A2 Blok D:6 Zonguldak - Turkey Postal code: 67630 drtarikakar@gmail.com

\section{SUMMARY}

Objective: Chronic hepatitis $\mathrm{C}(\mathrm{CHC})$ continues to be a critical problem. The liver fibrosis score is the most valuable tool in determining treatment and prognosis. Liver biopsy is still considered a gold method but, due to unmet needs, new non-invasive markers are required. The aim of this study was to investigate any possible relationship between serum angiotensin-converting enzyme (ACE) levels and the stages of liver fibrosis in patients with CHC.

Method: A total $100 \mathrm{CHC}$ and 100 healthy subjects were enrolled in this study. The relationship between serum ACE level and the stages liver fibrosis was investigated using three different formats, as follows: (group [G]-I, classic Ishak's Score from F1 to F6; G-II, mild [F1-2], moderate [F3-4] and severe [F5-6]; G-III, mild [ $\leq \mathrm{F} 2]$ and advanced [F > 2]). The clinical usability of serum ACE level for both groups was also investigated.

Results: Median serum ACE levels were higher in the healthy group than in $\mathrm{CHC}$ (42.5 [7-119] vs. 36 [7-91] U/I, $\mathrm{p}=0.002)$. There was no statistical difference among the three different fibrosis groups (G-I, G-II, G-III, $p=0.797$, $p=0.986$, and $p=0.874$ ) and no correlation between serum ACE level and the stages of liver fibrosis $(\mathrm{r}=0.026, \mathrm{p}=0.923)$. The usability of serum ACE for evaluated patients with CHC and healthy subjects were calculated as $47 \%$ and $64 \%$, respectively.

Conclusion: Our study indicated that there is no relationship or correlation between serum ACE levels and stages of liver fibrosis in patients with $\mathrm{CHC}$. The assessment of serum ACE level using genetically corrected reference values may provide more accurate results.

Keywords: Hepatitis C, Chronic. Peptidyl-Dipeptidase A. Liver Cirrhosis.

\section{INTRODUCTION}

Chronic hepatitis $\mathrm{C}(\mathrm{CHC})$ infection continues to be a serious problem worldwide despite the advent of new direct-acting antiviral therapy and increased public and individual awareness of the hepatitis $\mathrm{C}$ virus. ${ }^{1,2}$ The burden of chronic hepatitis $\mathrm{B}$ virus infection that was the foremost cause and the most important agent in the past decade is decreasing as expected due to the use of efficient and well-organized national vaccination programs and treatment, while the burden of $\mathrm{CHC}$ is increasing even now due to expensive new generation treatments and lack of the preventive vaccination. ${ }^{1-3}$ In the decision-making process of a patient newly diagnosed with $\mathrm{CHC}$, ascertaining the stage of liver fibrosis is one of the most important actions in the selection of treatment options and predict- ing long-term outcomes. ${ }^{3,4}$ Consequently, the stage of liver fibrosis is also accepted as the most valuable and well-considered marker in the context of important guidelines such as The European Association for the Study of the Liver (EASL) and American Association for the Study of Liver Diseases (AASLD). ${ }^{5,6}$

For a better understanding of current liver condition regarding fibrosis stage and other related etiologies, liver biopsy is still considered the gold standard method. There is, however, a significant amount of needs unmet using this technique, such as the very reduced size of the biopsy sample, which is not representative of the entire liver, sampling or intraobserver errors, and invasive procedure complications including risk of minor or major bleeding, or death. In addition, liver biopsy may be con- 
traindicated or unfeasible due to physical anomalies (namely, the ribs), thrombocytopenia, and prolonged prothrombin time..$^{7-9}$ Given these limitations, non-invasive methods or markers are needed as an alternative to liver biopsy and are currently under intense scrutiny. ${ }^{10}$ A non-invasive method should be easy to perform, inexpensive and yield reliable, reproducible results that can characterize early and/or advanced fibrosis. Many markers, measurements and methods have been evaluated so far for this aim, but none was able to prevail over liver biopsy for liver fibrosis evaluation. ${ }^{11}$ Moreover, the performance and usability of these methods are claimed to be good, but many do not produce a stricking effect due to poor study design and an insufficient number of patients and/or controls. ${ }^{12}$

The measurement of circulating angiotensin-converting enzyme (ACE) levels is one of the mentioned methods promising some good results in the early studies. ${ }^{13}$ The idea of measuring serum ACE levels to point out the stage of liver fibrosis dates back to the studies concerning the role played by renin-angiotensin-aldosterone (RAS) axis and angiotensin in liver fibrosis. ${ }^{14}$ In these studies, the RAS system and related factors such as angiotensin I, II and serum ACE were claimed to be the key elements affecting and regulating liver fibrosis. At the same time, the effect of some drugs that block the RAS system on liver fibrosis has been investigated. ${ }^{15,16}$ However, a few studies have been conducted on the importance of serum ACE level during the stage of liver fibrosis before until now. Up to today, the measurement of serum ACE level for liver fibrosis has only been studied in the context of hepatitis $\mathrm{B}$ and autoimmune hepatitis, with promising results according to the authors. ${ }^{17,18}$ However, in these studies, the value of serum ACE could not be assessed in deepth due to the insufficient number of patients and controls, as well as a lack of studies on usability. Many patients with chronic liver disease are usually detected and treated later in life and so plenty of comorbidities that often affect the serum ACE level can exist. Despite a lack of data regarding the issue, many patients initially presumed eligible for these studies are ultimately excluded on the account of diseases that affect their serum ACE levels. In this study, we investigated the importance and suitability of serum ACE level as a marker of liver fibrosis in a relatively large cohort group including $\mathrm{CHC}$ patients and a healthy control group.

\section{Method}

This study was designed at a reference and research center for liver diseases, and was approved by the local ethics committee on 6/29/2016 as clinical trial no. 2016-79$29 / 06$. The patients' files were first reviewed retrospectively to determine suitability for this study between May 2010 and May 2016. In brief, a total of 213 hepatitis C patients were searched, but 79 and 34 of all patients were excluded from the study due to chronic diseases affecting serum ACE levels and the deficiency of diagnostic data, respectively. 156 dyspeptic but otherwise healthy subjects were evaluated in order to compose a control group. Of these, 56 were excluded for presenting conditions or minor illnesses that could affect their serum ACE levels. Thus, the study and control groups comprised 100 hepatitis $C$ patients and 100 healthy subjects, respectively. To be part of the study group, patients were required to present positive anti-HCV antibodies and detectable levels of HCV RNA, as well as yield a proper liver biopsy sample for evaluation of liver fibrosis, and blood samples for measurement of serum ACE levels using a specific commercial kit. Plasma anti-HCV antibody was tested using Abbott AxSYM Anti-HCV 3.0 (Abbott Laboratories, Germany) and HCV RNA levels were measured by using COBAS TaqMan HCV RNA assay, version 2.0 (Roche Diagnostics Systems Inc, USA ), with a lower limit of detection of 10 IU per milliliter. Serum ACE activity was measured by observing the alteration in absorbance at $340 \mathrm{~nm}$ of the hydrolysis of furylacrylolylphenylalanylglycylglycine (FAPGG) to FAP and GG (Sigma-Aldrich, Poole, UK) using an analyzer (Roche MIRA Analyser; Roche Diagnostic Systems, Welwyn Garden City, UK). Liver biopsy specimens were accepted eligible if they included 10 or more complete portal tracts, had more than $20-25 \mathrm{~mm}$ in length, were stained with Masson's trichrome and reticulin dyes, and were evaluated according to modified Ishak's scoring system (1995). The stage of fibrosis was stratified into tree groups as following: (group [G]-I, classic modified Ishak's Score from F1 to F6; group-II, mild [F1-2], moderate [F3-4] and severe [F5-6]; group-III, mild [ $\leq \mathrm{F} 2]$ and advanced [F>2]).

Some of the patients with conditions that might affect the serum ACE level were excluded from the study. These conditions were accepted as following; 1 ) hypertension; 2 ) receiving any antihypertensive or other drugs that might potentially interact with the RAS system such as ACE inhibitors; 3) diabetes mellitus; 4) any renal parenchymal diseases; 5) sarcoidosis; 6) acute or chronic inflammation with elevated C-reactive protein or sedimentation; 7) concomitant chronic liver disorders; 8) moderate or severe cardiopulmonary problems. All CHC patients were categorized as Child-Plug grade $\mathrm{A}$ and, as expected, those with Child-Plug grade B and C as well as esophageal varices or any signs of decompensated 
cirrhosis were excluded. The healthy control group was equivalent to the patient group regarding age and gender and consisted of those who did not have chronic health problems other than dyspeptic complaints. Serum ACE levels were obtained from healthy controls using the same method as for the CHC patient group. The study was performed in agreement with the guidelines of the Helsinki declaration.

\section{Statistical analysis}

Statistical analyses were conducted using SPSS 19 (Chicago, Illinois, USA). Patient and control groups were investigated in terms of distribution, frequency and the difference between the two groups. A p-value lower than 0.05 was accepted as statistically significant. Gender, age and serum ACE levels were investigated using Chi-square tests, Student's t-test and Mann-Whitney U test, respectively. Spearman's correlation coefficient was used to evaluate the correlations between serum ACE levels and liver fibrosis stages. Normally distributed data are demonstrated as means and standard deviation (SD), while non-normally distributed data are represented by median and minimum-maximum.

\section{Results}

A total of 100 patients with hepatitis $C$ and 100 dyspeptic healthy subjects were included in this study. Clinical suitability and usability of serum ACE level were calculated as $47 \%$ for $\mathrm{CHC}$ group and $64 \%$ for healthy subjects. The median age was 56.5 (36-73) years for the CHC group and 55 (35-79) years for the healthy subjects. There were 30 females and 70 males in the CHC group and 40 females and 60 males in the healthy group. There was no statistically substantial difference in both groups regarding age and sex. The important demographic data are illustrated in Table 1. The median serum ACE level was higher in the healthy group than in patients with $\mathrm{CHC}$ (42.5 [7-119] vs. 36 [7-91] U/I, p=0.002) (Figure 1). The proportion of patients in terms of different fibrosis stages was calculated as follows: group-I was $\mathrm{F} 1=5(5 \%)$, $\mathrm{F} 2=17(17 \%), \mathrm{F} 3=31$ (31\%), F4=18 (18\%), F5=22 (22\%) and $\mathrm{F} 6=7(7 \%)$. The group-II was mild $(\mathrm{F} 1-2)=22(22 \%)$, moderate $(\mathrm{F} 3-4)=48(48 \%)$, and severe $(\mathrm{F} 5-6)=29(29 \%)$, and the group-III was mild $(\mathrm{F} \leq 2)=22(22 \%)$ and advanced $(\mathrm{F}>2)=78$ (78\%). There was no statistically significant difference among the different fibrosis groups regarding serum ACE levels (Figure 2). There was no correlation between the serum ACE and HAI, MELD and the scores of fibrosis $(r=-0.058, r=-0.121, r=-0.026$ and $p=0.567$, $\mathrm{p}=0.231, \mathrm{p}=0.923$, respectively).

\section{Discussion}

CHC is still considered a major problem all over the world. When choosing the optimal and the best treatment modality for these patients, the stage of liver fibrosis is accepted to be the key element, according to EASL and AASLD guidelines. ${ }^{5,19}$ For instance, some new and effective drugs are used in CHC patients with early fibrosis, but are contraindicated to $\mathrm{CHC}$ patients with advanced fibrosis. ${ }^{20,21}$ As mentioned in the introduction, liver biopsies are still considered the best method for detecting liver fibrosis. However, due to unmet needs, a growing number of non-invasive markers has been suggested for prediction of the stage of liver fibrosis..$^{10}$ Also, there is an increasing number of ongoing studies for this purpose. ${ }^{22}$ Measuring serum ACE levels is one of them, showing promising results in early studies, although some paradoxical outcomes have also been reported recently. ${ }^{16-18,23}$ The issue of non-invasive methods has increasingly attracted many researchers, and frequently new studies on the topic are added to the literature.

In the present study, we investigated the relationship between serum ACE levels and the stage of liver fibrosis in a relatively large cohort including $100 \mathrm{CHC}$ patients and 100 healthy control subjects. Interestingly, our results are different from previous studies due to the following points. To our knowledge, this is the first study to investigate the relationship between serum ACE level and the stages of liver fibrosis in patients with $\mathrm{CHC}$, as well as the

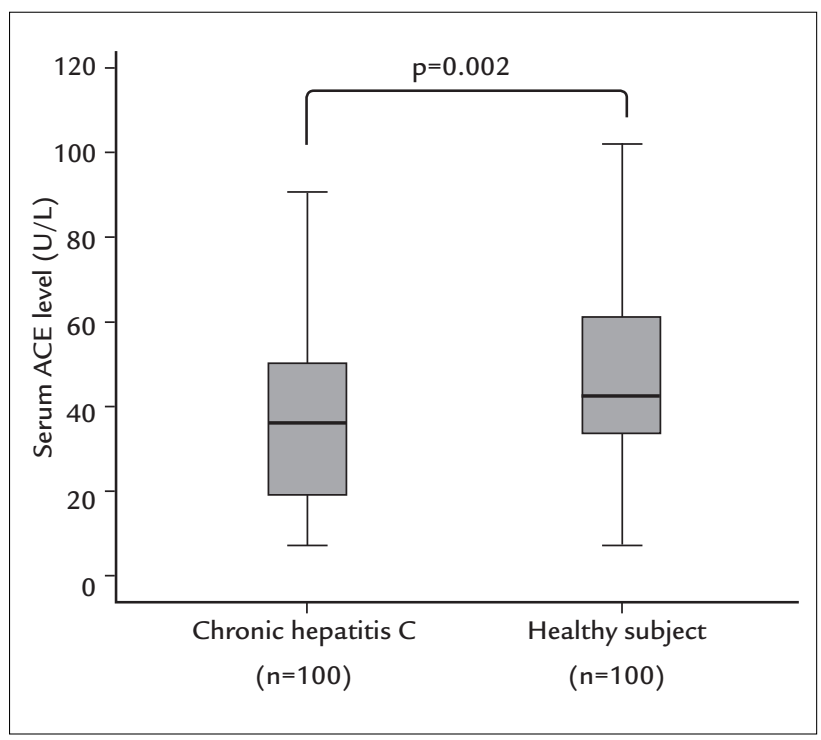

FIGURE 1 The median serum ACE level was higher in the healthy group than in patients with chronic hepatitis C (42.5 [7-119] vs. 36 [7-91] U/L, $P=0.002$ ) (95\% confidence interval and median values are shown). 


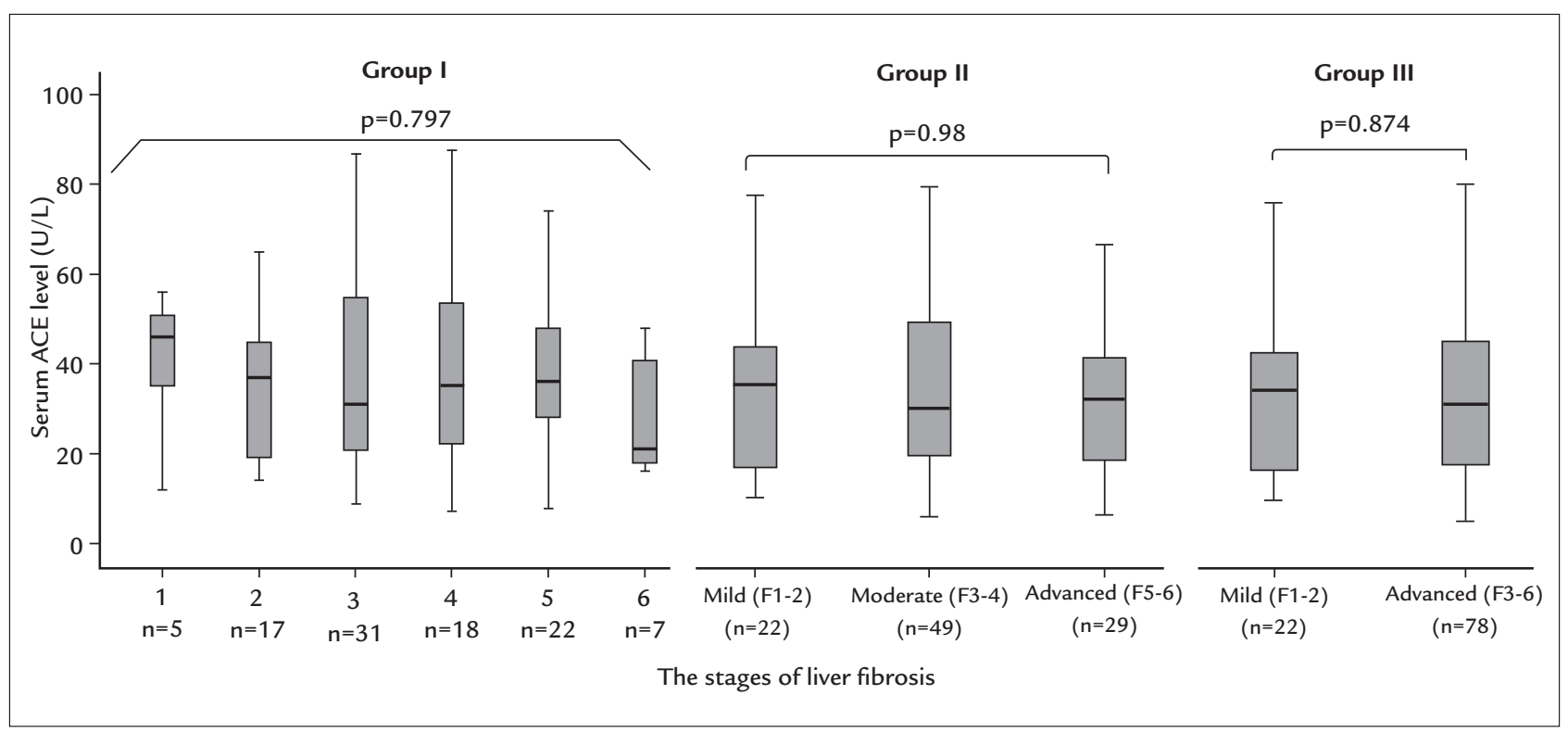

FIGURE 2 The median serum ACE level was not different statistically among groups I, II and III and there was also no relationship between serum ACE levels and the different liver fibrosis stages (95\% confidence interval and median values are shown).

TABLE 1 Important characteristics of the study population.

\begin{tabular}{|c|c|c|c|}
\hline & Chronic hepatitis C patients $(n=100)$ & Healthy group $(n=100)$ & $\mathbf{p}$ \\
\hline Age (years [min-max]) & $56.5(36-73)$ & $55.0(35-79)$ & 0.082 \\
\hline Gender (n [\%]) & & & 0.138 \\
\hline Male & $30(30 \%)$ & $40(40 \%)$ & \\
\hline Female & $70(70 \%)$ & $60(60 \%)$ & \\
\hline Blood pressure $(\mathrm{mmHg})$ & & & NS \\
\hline Systolic & $112 \pm 18$ & $121 \pm 22$ & \\
\hline Diastolic & $62 \pm 13$ & $71 \pm 13$ & \\
\hline Creatinin (mg/dL) & $0.97 \pm 0.2$ & $0.88 \pm 0.1$ & NS \\
\hline Serum ACE (U/L) (median [min-max]) & $36(7-91)$ & $42.5(7-119)$ & 0.002 \\
\hline $\mathrm{HAl}$ & $10 \pm 3.2$ & & \\
\hline MELD & $7 \pm 1.3$ & & \\
\hline $\operatorname{AFP}(n g / m L)$ & $4.1 \pm 3.4$ & $3.1 \pm 1.9$ & NS \\
\hline T.Bil. $(\mathrm{N}<1.2 \mathrm{mg} / \mathrm{dL})$ & $0.97 \pm 0.17$ & $0.85 \pm 0.1$ & NS \\
\hline Child-Plug Score & $A(100 \%)$ & & \\
\hline
\end{tabular}

suitability and usability of serum ACE levels in patients with chronic liver disease. According to the literature, a non-invasive marker to be used for detecting of the stage of liver fibrosis should be easy to perform, inexpensive and yield reliable, reproducible results, as well as remain unaffected by other chronic diseases and medication. Unfortunately, the levels of serum ACE could be affected by many drugs and chronic diseases that are commonly present in most of the CHC patients. Consequently, we researched this issue and finally found that the usability of serum ACE in our patients with $\mathrm{CHC}$ was only $47 \%$. This means that serum ACE may not be a suitable non-invasive marker for all CHC patients. In addition, studies on serum ACE levels and liver fibrosis usually include a relatively small number of the patients and healthy controls. ${ }^{17,18}$

Also, contrary to other studies, we found a higher level of serum ACE in the healthy controls (42.5 U/L) compared to the patients with CHC (36 U/L), and no correlation between serum ACE and the stages of liver fibrosis. In previous studies, the most striking results 
regarding the relationship between serum ACE and the stage of liver fibrosis have been found first by Purnak et al. ${ }^{17}$ and Efe et al. ${ }^{18}$ in patients with chronic hepatitis B and autoimmune hepatitis, respectively. Interestingly, both authors found nearly the same results that serum ACE was higher in the patient groups compared to the healthy control groups (hepatitis B study results: chronic hepatitis B vs. healthy control, 48.4 [14-83] U/L vs. 26.2 [12-48] U/L, $\mathrm{p}<0.001$, and autoimmune hepatitis study: autoimmune hepatitis vs. healthy control, 58 [38-142] vs. 34.5 [10-59] $\mathrm{p}<0.001) .{ }^{17,18}$ Moreover, they both found a significant correlation between serum ACE and the stages of liver fibrosis, and identified an optimum serum ACE cutoff level for advanced fibrosis as $52.5 \mathrm{U} / \mathrm{L}$ and $65 \mathrm{U} / \mathrm{L}$ with high specificity and sensitivity. ${ }^{17,18}$ Based on data, Efe et al. ${ }^{18}$ claimed that serum ACE might also be an attractive inflammation marker due to the fact that serum ACE levels increase proportionally with the severity of the interface hepatitis. When these two studies are considered together, the most critical problem is that the number of researched patients and control group is very small (Purnak et al. ${ }^{17}$ included 50 chronic hepatitis B patients vs. 20 healthy controls, and Efe et al. ${ }^{18}$ had 73 patients with autoimmune hepatitis vs. 32 healthy controls); some patients in the study group had signs of cirrhosis. Also, these results are not confirmed in a relatively wide range of studies performed by Turhan et al. ${ }^{23}$ as well as our study.

A similar discrepancy that has been noticed in some of the drug studies refers to blockage of RAS. In early studies on the latter, despite a reduction in liver fibrosis with the use of some ACE inhibitors, the results were not confirmed by any new studies performed with lisinopril, known as one of angiotensin-converting enzyme inhibitors. ${ }^{14,24,25}$ Lisinopril has shown no major histomorphological alterations in regenerating fibrotic liver tissues and has a beneficial effect on the regression of liver fibrosis. ${ }^{16}$ These conflicting results may also be explained by the lack of consideration of the polymorphism of ACE gene in the study population. Polymorphism of the ACE gene has the greatest impact on serum ACE levels with a strong genetic influence and large interindividual differences. ${ }^{26}$ Studies investigating I/D polymorphism in the ACE gene have demonstrated that, while individuals with D allele have advanced ACE activity, individuals with I allele have lower ACE activity than those with D allele. ${ }^{27}$ Moreover, the ACE gene polymorphism can cause a considerable amount of changes, up to $28 \%$ increase in serum ACE levels, depending on the ethnic background of the study population. ${ }^{28}$ Consequently, the interpretation of serum ACE values without regard to ACE gene polymor- phism may cause a masking effect regarding the exact values. ${ }^{23}$ Based on these conflicting results, we suggest that serum ACE levels should be interpreted in view of the polymorphism of ACE gene and used with genotypecorrected reference values.

\section{Conclusion}

In conclusion, our results showed that the measurement of serum ACE for assessment of the stage of liver fibrosis is not usable and suitable in patients with CHC. Also, there is no correlation between serum ACE levels and the stages of liver fibrosis. Based on recent research, it is not possible to interpret the level of serum ACE correctly without considering the polymorphism of the ACE gene. This hinders the use of serum ACE as an easy, cost-effective and reliable marker of liver fibrosis.

\section{References}

1. Hézode C. Pan-genotypic treatment regimens for hepatitis C virus: advantages and disadvantages in high- and low-income regions. J Viral Hepat. 2017;24(2):92-101.

2. Dhiman RK, Satsangi S, Grover GS, Puri P. Tackling the hepatitis C disease burden in Punjab, India. J Clin Exp Hepatol. 2016;6(3):224-32.

3. Carmona I, Cordero P, Ampuero J, Rojas A, Romero-Gómez M. Role of assessing liver fibrosis in management of chronic hepatitis $\mathrm{C}$ virus infection. Clin Microbiol Infect. 2016;22(10):839-45.

4. Potts JR, Maybury CM, Salam A, Barker JN, Agarwal K, Smith CH. Diagnosing liver fibrosis: a narrative review of current literature for dermatologists. $\mathrm{Br}$ J Dermatol. 2016;177(3):637-44.

5. European Association for the Study of the Liver. EASL Recommendations on treatment of hepatitis C 2016. J Hepatol. 2017;66(1):153-94.

6. Jacobson IM. The HCV treatment revolution continues: resistance considerations, pangenotypic efficacy, and advances in challenging populations. Gastroenterol Hepatol (N Y). 2016;12(10 Suppl 4):1-11.

7. Lidbury JA. Getting the most out of liver biopsy. Vet Clin North Am Small Anim Pract. 2017;47(3):569-83.

8. Chi H, Hansen BE, Tang WY, Schouten JN, Sprengers D, Taimr P, et al. Multiple biopsy passes and the risk of complications of percutaneous liver biopsy. Eur J Gastroenterol Hepatol. 2017;29(1):36-41.

9. Lesmana CR, Cahyadinata L, Pakasi LS, Lesmana LA. Efficacy of prothrombin complex concentrate treatment in patients with liver coagulopathy who underwent various invasive hepatobiliary and gastrointestinal procedures. Case Rep Gastroenterol. 2016;10(2):315-22.

10. Boursier J, Ledinghen V, Leroy V, Anty R, Francque S, Salmon D, et al. A stepwise algorithm using a at-a-glance first-line test for the non-invasive diagnosis of advanced liver fibrosis and cirrhosis. J Hepatol. 2017;66(6):1158-65.

11. Karanjia RN, Crossey MM, Cox IJ, Fye HK, Njie R, Goldin RD; et al. Hepatic steatosis and fibrosis: non-invasive assessment. World J Gastroenterol. 2016;22(45):9880-97.

12. Stasi C, Milani S. Non-invasive assessment of liver fibrosis: between prediction/prevention of outcomes and cost-effectiveness. World J Gastroenterol. 2016;22(4):1711-20.

13. Borowsky SA, Lieberman J, Strome S, Sastre A. Elevation of serum angiotensin-converting enzyme level. Occurrence in alcoholic liver disease. Arch Intern Med. 1982;142(5):893-5.

14. Kardum D, Fabijanić D, Lukić A, Romić Z, Petrovecki M, Bogdanović Z, et al. Correlation of endothelin-1 concentration and angiotensin-converting enzyme activity with the staging of liver fibrosis. Coll Antropol. 2012;36(2):413-8.

15. Toblli JE, Ferder L, Stella I, Angerosa M, Inserra F. Enalapril prevents fatty liver in nephrotic rats. J Nephrol. 2002;15(4):358-67.

16. Ambreen A, Jahan S, Malik S. Effect of angiotensin-converting enzyme inhibitor, lisinopril on morphological and biochemical aspects of fibrotic liver regeneration. Saudi J Gastroenterol. 2016;22(6):428-34. 
17. Purnak T, Beyazit Y, Oztas E, Yesil Y, Efe C, Torun S, et al. Serum angiotensinconverting enzyme level as a marker of fibrosis in patients with chronic hepatitis B. J Renin Angiotensin Aldosterone Syst. 2012;13(2):244-9.

18. Efe C, Cengiz M, Kahramanoğlu-Aksoy E, Yilmaz B, Özșeker B, Beyazt Y, et al. Angiotensin-converting enzyme for noninvasive assessment of liver fibrosis in autoimmune hepatitis. Eur J Gastroenterol Hepatol. 2015;27(6):649-54

19. AASLD/IDSA HCV Guidance Panel. Hepatitis C guidance: AASLD-IDSA recommendations for testing, managing, and treating adults infected with hepatitis C virus. Hepatology. 2015;62(3):932-54.

20. Perelló C, Carrión JA, Ruiz-Antorán B, Crespo J, Turnes J, Llaneras J, et al; Spanish Collaborative Group for the Study of the Use of Hepatitis C DirectActing Drugs. Effectiveness and safety of ombitasvir, paritaprevir, ritonavir \pm dasabuvir \pm ribavirin: An early access programme for Spanish patients with genotype $1 / 4$ chronic hepatitis $C$ virus infection. J Viral Hepat. 2017;24(3):226-37.

21. Omata M, Kanda T, Wei L, Yu ML, Chuang WL, Ibrahim A, et al. APASL consensus statements and recommendation on treatment of hepatitis C. Hepatol Int. 2016;10(5):702-26.

22. Stasi C, Milani S. Evolving strategies for liver fibrosis staging: non-invasive assessment. World J Gastroenterol. 2017;23(2):191-6.
23. Turhan NK, Ilikhan SU, Hamamcioglu AC, Ustundag Y, Dursun A, Kokturk F. Angiotensin-converting enzyme gene polymorphism (insertion/deletion) and liver fibrosis in Turkish patients from the western Black Sea region, Turkey. Genet Mol Res. 2015;14(4):17079-90.

24. Yoshiji H, Kuriyama S, Fukui H. Blockade of renin-angiotensin system in antifibrotic therapy. J Gastroenterol Hepatol. 2007;22(Suppl 1):S93-5.

25. Yoshiji H, Kuriyama S, Noguchi R, Yoshii J, Ikenaka Y, Yanase K, et al. Combination of interferon-beta and angiotensin-converting enzyme inhibitor, perindopril, attenuates the murine liver fibrosis development. Liver Int. 2005;25(1):153-61

26. Biller H, Zissel G, Ruprecht B, Nauck M, Busse Grawitz A, Müller-Quernheim J. Genotype-corrected reference values for serum angiotensin-converting enzyme. Eur Respir J. 2006;28(6):1085-90.

27. Camós S, Cruz MJ, Morell F, Solé E. Genetic-based reference values for angiotensin-converting enzyme (ACE) according to I/D polymorphism in a Spanish population sample. Clin Chem Lab Med. 2012;50(10):1749-53.

28. Tiret L, Rigat B, Visvikis S, Breda C, Corvol P, Cambien F, et al. Evidence, from combined segregation and linkage analysis, that a variant of the angiotensin I-converting enzyme (ACE) gene controls plasma ACE levels. Am J Hum Genet. 1992;51(1):197-205. 